\title{
Inhaltsverzeichnis Jahrgang 14
}

$\begin{array}{ccrrrr}\text { Heft 1: Seiten } & 1 \text { bis } & 50 \\ , & 2: & , & 51 & , & 90 \\ ", & 5: & , & 91 & , & 150 \\ , " & +: & , & 151 & , & 170\end{array}$

\author{
Heft 5: Seiten 171 bis 218 \\ , 6: , , $219,26+$ \\ , 7: , 265,306 \\ , 8: $, \quad 307,3+6$
}

\section{Verfasserverzeichnis}

\section{$(\mathrm{KM})=$ Kurze Mitteilung}

Alföldi, J., und Paštcka, M., Ozonisierung der Acetylcellulosen mit verschiedenem Acetylierungsgrad (KM) 389

Bara, II., Substitutionsreaktionen am Caprolactam und Cyclo-di-aminocaproyl. Synthese von Lysin aus Cyclo-di-aminocaproyl . . . . . כ6

Barnitzke, $W$., und Georgc, J., Me. thode zur Bestimmung der Fadengeschwindigkeit bei der Frspinumg von Regeneratfïden (KM) . . . .

Bartels, U., und Hoyme, HI., Zur Reinheitskontrolle von Natrium. Allylsulfonat und seiner Bestim. mung neben anderen monomeren Acrylverbindungen. 4. Mitt. zur Analytik der Polyacrylnitrilfasern (K.M) j.

Barth, R., Winkler, F., und Sommer, $I I$., Lntersuchungen über das $\mathrm{Kli}$ maverhalten von Textilien. II. Die Veränderung des \%ugfestigkeitsverhaltens von Geweben aus verschiedenen Faserstoffen durch Klimaeinfliusse

Barthel, R., Schater, G., und Hof. mann, H., Schwingungsuntersuchungen an Textilspindeln

Bartsch, I)., Schmiedeknecht, H., und Gohllic, B. t, Der Xanthogenierungsgrad von Eintopfviskosen . 351

Baudisch, J., und Philipp, B., Zum Finfluß des Gelteilchengehaltes vor Yiskosespimlimsungen auf den ïbermolekularen Bau daraus hergestellter Cellulose-Regeneratfïien .

Bobeth, $H^{\prime}$., und Rauc, P., Zur Bestimmung von Festigkeits. und Formänderungseigenschaften an Glasgeweben

- , Heger, A., und Münch, H., Bestimmung von Avivagemenge und Avivagegleichmäßigkeit auf Iiskoseseiden mittels ${ }^{35} \mathrm{~S}$-markierter Avivage

-, Schönc, A., und Piechottka, G., Zur mikroskopischen und makroskopischen Qualitätsbeurteilung von Polyesterfaserstoffen

-, und Vollrath, L., Zum Ieformationsverhalten von Faserstoffen bei Quetschbeanspruchung. . . . 431, Boróczy, E., Das Streichgarnspinnen an Ringspinnmaschinen mit Dreh. röhrchen-Streckwerk Teil I u. JI

$$
75 \text {, }
$$

Casperson, G., Maron, R., Gröbc, A., und Klarc, II., Versuche zur Untersuchung der koagulierenden Wirkung von Viskosespimmbädern. 18. Witt. uber die Fadenbildung von Viskosekapillarfäden

Čirlič, J. B., Theorie und Praxis der Mischungen von Wolle und Chemiefasern. V. Repräsentationsqualität, Dauerhaltbarkeit, physiologische Eigenschaften und Griff der Misch- gewebe aus Wolle und Viskose-Zellwolle . . . . . . . . . . . . . -, und Malkoci, O., Studie über den Einfluß 3 der Polyesterfaser in Drei- und Zweikomponenten-Misch. geweben mit Wolle und Viskose. Zellwolle . . . . . . . . . . 55 Claus, $H^{*}, \quad$ Gohlke, $B .+$ und Schiniedeknccht, II., Der Abbau der Cellulose bei der Herstellung von Fintopfviskosen

-, und Schmicdeknecht, H., Zur lieaktion zwischen Alkalilauge und Schwefelkohlenstoff (K.Vl)

Cypryk, J., Eaczkoucsi, M., und Picchucki, S., Fasern aus Nischungen von Polyacrylnitril und Produk. ten seiner Hydrolyse sowic anderen Hochpolymeren.

Dcmus, If., Zur Entziindlichkeit von Cellulose (K.MI)

Deters, $W_{.}$, und Grohn, $H$., Über die Kinetik des mechanochemischen 47+ Abbaus von Cellulose und Cellulose triacetat durch Schwingmahlung. 2. Mitt. zur Uechanochemie der 397 Cellulose und Cellulosederivate

-, und Huang, Dehja-chian, Mechanochemische Darstellung von Block. und Pfropfpolymeren aus Cellulosetriacetat und Vinylchlorid durch Schwingmahlung. 5. Mitt. zur Mechanochemie der Cellulose und Cellulosederivate . . . . . . . . 18

Feldman, D., Vasiliu, C., und Simionescu, C., Die Pfropfung von Polyacrylnitril auf Cellulose

Frenzel, $W$., Papierfaden. . . . . 4

16 Fritzsche, $P$., und Ulbricht, J., Das Redoxsystem Peroxydisulfat-Hydrogensulfit-Metallionen als Initiator der Acrylnitril-Polymerisation.

Teil I: Einfluß des primären Salz. effektes . . . . . . . . . . . 320 -, 一, Teil II: Der Mechanismus des Redoxsystems PeroxydisulfatHydrogensulfit-Iisen(II)-Ionen . .

Galanský, M., und Lacko, V., Die Jodsorption der Polyesterfasern, ein 489 Maß für die Gleichmäßigkeit der Fixierung

Gchrkc, K., Beiträge zum Reaktionsmechanismus der Schnellpolymerisation des Caprolactams. II. Bestimmung der cyclischen Oligomeren der $\varepsilon$-Aminocapronsäure (KN) . . . 359

,-- . III. Lntersuchungen zur Startreaktion

George, J., und Barnitzke, $W^{\prime}$., Me. 313 thode zur Bestimmung der Faden. geschwindigkeit bei der Erspinnung von Regeneratfäden (K.ll) . . . . Gillert, H., und Neumann, P., Lini. ge Modelle für Lebensdauerverteilungen und ihre slatistische Priifung
Gohlke, B. t, Schmicdeknecht, II., 26 und Claus, $W$., Der Abbau der Cellulose bei der Herstellung von Eintopfviskosen . . . . . . . .

-, Schmiedeknecht, H., und Bartsch, D., Der Xanthogenierungsgrad vol 537 Eintopfviskosen . . . . . 351 Grimm, II., Zur Fixiergradbestim. mung an Polyesterseiden (KM) 81, 390

51 Gröbe, A., Pekala, T., Jost, H., Maron, R., und Klare, H., Lintersuchungen zum Ionenaustausch ail Zinkcellulosexanthogenat. 17. Mitteilung über den Fadenbildungsmechanismus von Viskosekapillarfäden . . . . . . . 131 -, Maron, R., Klare, H., und Casperson, G., Versuche zur Lntersuchung der koagulierenden Wirkung von Viskosespimmbädern. 18. Mitt.

549 - über den Fadenbildungsmechanis. mus von Viskosekapillarfäden . . . 315

-, Purz, H.-J., Maron, R., und Klare, H., Lntersuchungen über die geordnete Koagulation von Na-cellulosexanthogenatlösungen. 19. Mitt.

58 ïber den Fadenbildungsmechanismus von Celluloseregeneratfäden . 347

-, Jost, II., und Klarc, II., Reaktionskinetische Lintersuchungen bei der Entstehung von Celluloseregeneratfiden. 20. Mitt, über den Fa. denbildungsmechanismus von V"isko-

185 sekapillarfäden (1. Teil) . . . . . 522

Gröbc, V., Sandner, H., und Rcichert, $I I$., Lntersuchungen über den 63 Fadenbildungsmechanismus beim 440 Naßspimen von Polyacrylnitril. -, und Versäumer, H., Über die Fadenbildung beim Schmelzspinnen (Teil I u. II) . . . . . . . 249, 288 Grohn, H., und Deters, $H^{\prime}$., Uber die Kinetik des mechanochemischen 520 Abbaus von Cellulose und Cellulose. triacetat durch Schwingmahlung. 2. Nitt. zur Mechanochemic der Cellulose und Cellulosederivate . . Gründig, K., und Richl, II.-J., Kammgarngewebe aus I)reifaser. mischungen . . . . . . . . . 202

68 Heger, A., und Sonntag, P., Die Anwendung von radioaktiven Strah. lenquellen in der Textilindustrie. Teil V. Die Anwendungsmöglichkeiten von $\beta$-Zählrohren . . . . . . - , Ein neues Verfahren zur Bestimmung der Menge und der Gleichmäßigkeit von Fremdsubstamzen (z. B. Avivage) auf 'T'extilfäden (K.I) . . . . . . 12 -, Bobeth, $\boldsymbol{H}^{\prime}$, und Münch, H., Bestimmung von Avivagemenge und Avivagegleichmäßigkeit auf Viskoseseiden mittels ${ }^{35} \mathrm{~S}$-markierter Avivage . . . . . . . . . . . 281 